\title{
Comment appliquer la matrice GDE ? Quelles adaptations aux facteurs de risque d'accidents des jeunes conducteurs novices?
}

\author{
Which pedagogical innovations in the improvement of the licensing process? How to apply the \\ GDE matrix? How is it adapted to the accident risk factors of young novice drivers?
}

\author{
Jean-Pascal Assailly \\ (C) IFSTTAR et Éditions NecPlus 2016
}

\begin{abstract}
Résumé Cet article présente une revue des diverses innovations pédagogiques apparues dans les pays industrialisés afin de réduire le sur-risque des jeunes conducteurs novices, ainsi que de leurs évaluations. On présente d'abord les logiques scientifiques et politiques qui ont conduit certains pays à se focaliser sur la diminution de l'exposition au risque (accès graduel à la conduite ou permis progressif) ou d'autres sur l'éducation du conducteur afin de renforcer ses diverses compétences lors de cette phase d'inexpérience. À partir du champ théorique et méthodologique impulsé à partir de la matrice GDE, on passe en revue les divers types de formation, avant le permis (apprentissage avec les enseignants de la conduite, conduite accompagnée, entraînement sur simulateur) et après (entraînements aux manœuvres de pilotage, formations cognitives, entraînement à la perception des dangers, conduite commentée, complètement de tâche, entraînements des «compétences de vie », programmes d'amélioration de l'autoévaluation). On conclura par les enseignements de cette revue à propos des bonnes pratiques visant à modérer la confiance excessive, en développant les compétences métacognitives (autoévaluation plus réaliste de soi) à chaque niveau de la matrice GDE
\end{abstract}

Mots clés formation - risque - jeunes conducteurs · méthodes pédagogiques compétences de vie

Summary This paper presents a review of the various innovations in driver training methods which have been elaborated in the industrialized countries, in order to reduce the over-risk of young novice drivers, and of their evaluations. First, we present the scientific and policy factors that have guided some countries to focus on the reduction of risk exposure (GDL or Graduated Driver Licensing) and other countries on driver education, in order to improve the various driving and safety skills

Jean-Pascal Assailly $(\varangle)$

Ifsttar, AME, LPC, F-78000 Versailles, France

e-mail : jean-pascal.assailly@ifsttar.fr during this phase of inexperience. Starting from the theoretical and methodological field of the GDE matrix, the various types of training are reviewed, before licensing (training with professional driving teachers, accompanied driving, simulator training) and after licensing (practical driving skills training, cognitive training like risk perception training, commented driving, task completion, life-skills training, self-evaluation training). Last, we conclude on what we have learnt about good practices concerning moderation of excessive confidence and development of meta-cognitive skills (more realistic self-evaluation) at each level of the GDE Matrix.

Keywords driver training $\cdot$ risk $\cdot$ young drivers $\cdot$ training methods $\cdot$ life-skills

\section{Introduction}

L'un des défis universels posé au secteur de la formation des conducteurs est le sur-risque accidentel des jeunes conducteurs novices : en 2014, les 18-24 ans constituent $8 \%$ de la population et $17 \%$ des tués ; après une baisse de $8,5 \%$ en 2014 essentiellement due à la baisse du nombre des jeunes motocyclistes accidentés, la mortalité des jeunes de 18-24 ans est en hausse de l'ordre de $7 \%$ en 2015 par rapport à 2014, essentiellement chez les piétons et les automobilistes [1]. Ceci nous rappelle que depuis 50 ans, l'accidentalité des jeunes suit toujours la tendance générale sur l'ensemble de la population [2].

Ce sur-risque s'observe dans tous les pays, donc dans des systèmes culturels, politiques, religieux, sociaux et réglementaires d'accès à la conduite très différents $[2,3,4]$ et depuis les débuts du recueil de données d'insécurité routière dans notre pays (1955). Cette constance du sur-risque partout et depuis toujours, quel que soit le système de formation mis en place, pourrait nous amener à conclure que la formation des conducteurs est impuissante à contrer ce phénomène. 
Par ailleurs, le sexe est un facteur encore plus fortement associé au sur-risque : ainsi, en 2014, $75 \%$ des tués sont des hommes. Sur la période courant de 18 à 40 ans, le sur-risque des hommes est maximal avec $80 \%$ de tués, et encore sur les deux femmes tuées sur 10, l'une l'est comme passagère d'un conducteur masculin. . En termes de responsabilité de l'accident mortel, le sexe est de loin le premier facteur de variation [5]. Au-delà des différences quantitatives, il existe aussi des différences qualitatives : les accidents des sujets féminins ressortent plus du registre de l'erreur, ceux des sujets masculins du registre de l'infraction. On peut donc être amené aussi à se demander si la formation peut faire quelque chose par rapport à cette énorme vulnérabilité masculine. . .

Un débat persiste depuis les débuts de la recherche en sécurité routière sur les importances respectives des deux facteurs du sur-risque des jeunes conducteurs novices, l'âge et l'inexpérience ; en effet, si l'âge était seul en cause (les caractéristiques biologiques, psychologiques et sociales de l'adolescence et de la post-adolescence, le décalage dans l'agenda neurobiologique entre la maturation du système de récompense des aires limbiques et celle du système de contrôle cognitif des décisions des aires préfrontales, la saillance des récompenses immédiates, la sensibilité aux influences des pairs, les effets des normes descriptives et injonctives, etc. [4]), la formation des conducteurs serait a priori peu opérante et efficace ; si l'inexpérience des jeunes conducteurs novices joue un rôle, la formation peut espérer avoir plus d'influence. Or, il semble bien que l'inexpérience joue également : les conducteurs novices ont un risque plus important d'accident dans les 6 premiers mois quel que soit leur âge [6], même si les jeunes conducteurs ont évidemment un risque plus important que les adultes novices.

On voit donc comment divers clivages épistémologiques organisent ce champ : jeunes/adultes, âge/inexpérience, masculin/féminin, infraction/erreur, etc. Toutefois, ces oppositions ne sont pas exclusives pour un même sujet.

Par ailleurs, les facteurs de risque sont si multiples et hétérogènes que nous sommes conduits à l'idée qu'une démarche éducative unique ne peut suffire pour résoudre ce problème. En effet, la connaissance accidentologique est suffisante maintenant pour la mise en évidence des facteurs de risque qu'une approche éducative doit tenter de traiter chez les jeunes conducteurs novices : en ce qui concerne l'âge, il y a la vitesse excessive, la conduite sous l'influence de l'alcool ou du cannabis, la distraction, la fatigue $^{1}$; en ce qui concerne l'inexpérience et les erreurs, on observe les déficiences de la perception des indices de danger, le désapprentissage après la sortie de l'auto-école, la

\footnotetext{
${ }^{1}$ Toutefois, on remarquera que ce sont aussi des facteurs de risque pour les conducteurs adultes expérimentés ! Si ce n'est ni l'âge ni l'expérience qui joue ici, nous pourrions nous demander si n'intervient pas un troisième facteur de confusion qui pourrait être la personnalité du conducteur!
}

variabilité interindividuelle du comportement, le comportement habituel et optimal.

\section{Les différences culturelles et sociétales à l'origine des approches visant la diminution de l'exposition au risque et/ou celle basées sur la formation}

Diverses stratégies ont été appliquées pour essayer de réduire ce sur-risque accidentel des jeunes conducteurs novices : en Amérique du Nord et en Australie, la stratégie dominante est de réduire l'exposition au risque lors de ces années d'inexpérience de la conduite par des systèmes dits « d'accès graduel à la conduite » ou de «permis progressif ». Dans ces pays où les transports en commun et la mobilité en deux-roues sont peu développés, les jeunes accèdent plus précocement à la conduite (entre 14 et 18 ans) car ils sont plus captifs et dépendants de la voiture. Cet accès graduel s'est fondé initialement sur une étude de 1971 en Caroline du Nord qui avait identifié la surreprésentation des conducteurs adolescents dans les accidents de nuit et lorsqu'un autre adolescent était le passager avant [7].

L'idée est de leur interdire au début un certain nombre de droits (conduite de nuit, avec des passagers, avec alcool, sur autoroute, etc.), puis au bout d'un certain nombre d'années (permis d'apprenti et probatoire), si le jeune n'est pas sanctionné pour avoir commis trop d'infractions et n'est pas impliqué dans des accidents, il obtient le permis de plein droit dans les délais prescrits (en Nouvelle-Zélande par exemple, les jeunes sortent de ce «parcours du combattant » à 25 ans...). Le jeune parcourt ainsi ses premiers milliers de kilomètres dans des situations de moindre risque puisque les facteurs de risque de l'accident mortel ont été ciblés (nuit, alcool, cannabis, fatigue, pression des passagers, vitesse, [2]).

Dans tous les pays où ce système d'accès graduel à la conduite a été évalué, on a observé effectivement des diminutions significatives des taux d'accidents des jeunes conducteurs novices (voir la revue de la littérature [8]) ; toutefois, l'accès graduel à la conduite n'abolit pas le sur-risque des jeunes conducteurs, et comme toutes les mesures de sécurité routière, même les meilleures, il finit par atteindre son asymptote d'efficacité et d'acceptabilité sociale ; on ne peut «enfermer » les jeunes pour les protéger, d'autres enjeux que l'insécurité routière existent pour cette cible (l'insertion, l'autonomie, la socialisation, etc.). L'accès graduel va par ailleurs être plus difficilement acceptable socialement dans les pays européens où l'accès au permis est plus tardif, alors que les jeunes sont majeurs.

En fait, l'une des raisons au développement de l'accès graduel à la conduite provient précisément des évaluations des formations des jeunes conducteurs réalisées dans les universités américaines dans les années 1980, et notamment 
de celle du programme du De Kalb County en Géorgie par la NHTSA (National Highway Traffic Safety Administration) : 16000 étudiants avaient été assignés de manière aléatoire à un programme d'entraînement de 20 heures (en classe, sur simulateur, sur route) ou à un groupe contrôle; si le programme avait amélioré les compétences de conduite, il ne se traduisait pas par un bénéfice préventif en ce qui concerne les accidents ; les sujets entraînés avaient même un taux supérieur d'accidents [9] car le programme produisait des accès au permis plus précoces et des expositions au risque plus importantes !

L'analyse de Lund [9] a été ensuite partiellement réfutée dans une analyse ultérieure des données du programme De Kalb par Smith (1994) [10] dans un rapport au Congrès qui notait une diminution de $6 \%$ des accidents, mais seulement pour la version courte du programme. Smith indiquait que « une seconde analyse du programme, tel qu'il a été développé, conduit, géré et évalué ne montre pas d'effets négatifs ». Ce programme a été mené aussi bien qu'il était possible sur le plan opérationnel. Donc, le problème provient de la manière par laquelle l'éducation des conducteurs novices est prodiguée aux États-Unis, c'est-à-dire « quand » et « comment ». Cette remarque de Smith (op. cit.) reste très pertinente aujourd'hui pour n'importe quelle application de formation des conducteurs novices.

Depuis, la majorité des collègues anglo-saxons avaient conclu que « l'éducation routière ne marche pas », que « la formation est contre-productive », ce qui a progressivement conforté la seule option de l'accès graduel et l'idée qu'il fallait protéger les jeunes malgré eux ! Aucune formation ne saurait contrer les effets d'une surexposition au risque du fait d'un accès plus précoce et de kilométrages plus importants, les pays à accès précoce devraient essayer de retarder l'accès et les pays à accès plus tardif de diminuer les kilométrages. On retrouve ici l'opposition bien connue entre « mieux conduire » ou « moins conduire » (veut-on baisser le risque relatif, le taux de jeunes tués au km parcouru, ou le risque absolu, le nombre total de jeunes tués, même si l'on ne change pas leur comportement mais en diminuant leur exposition au risque ?). Cette question revient souvent à propos du débat sur l'abaissement de l'âge d'accès : il permet d'augmenter la durée de l'apprentissage mais il augmente aussi l'exposition au risque...

Toutefois, cette position nord-américaine initiale est en train d'évoluer actuellement, du fait de résultats n'allant pas dans le même sens, vers une complémentarité entre accès graduel et formation initiale des jeunes conducteurs novices : par exemple, une étude épidémiologique récente ([11] a recensé tous les conducteurs adolescents dans le Nebraska (151 880 adolescents, 48,6 \% de filles, $51,4 \%$ de garçons) au cours d'une période de huit ans de 2003 à 2010 ; les conducteurs avec formation avaient significativement moins d'accidents (tués et blessés), d'infractions pour alcoolémie et d'infractions routières en général que les conducteurs sans formation, pendant les deux premières années de leur permis d'apprenti, donc à une période très importante de sur-accidentalité. Toutefois, cette étude à grande échelle n'a pas surmonté deux des biais méthodologiques les plus courants en ce qui concerne la validité des travaux évaluant les formations des conducteurs : le biais de sélection de l'échantillon (ce peut être les jeunes conducteurs les plus prudents qui choisissent de faire la formation), le biais d'exposition (le kilométrage annuel et les conditions de la conduite doivent être mesurés si l'on veut comparer deux populations de jeunes conducteurs).

Nous allons donc vers un rapprochement des positions européennes et nord-américaines, vers un consensus sur la nécessité d'une association entre accès graduel et formation. La question qui subsiste est celle-ci : quel type de formation des conducteurs avait été évalué dans ces années 1980 pour conclure que la formation est inefficace ? S'il s'agissait de formations uniquement centrées sur le pilotage alors que les facteurs de risque sont plutôt situés dans les styles de vie, il n'est pas surprenant d'avoir observé peu d'effets préventifs ! Comme si l'on voulait évaluer l'influence de cours de natation sur la performance en hockey sur glace ! Ce problème se pose aussi à propos de l'évaluation de l'efficacité préventive des formations dispensées actuellement dans les auto-écoles !

Inversement, de faibles compétences de conduite pourraient aussi être liées à des traits de personnalité qui pourraient être associés à un déficit des comportements de protection de soi, comportements communs à un style de vie dangereux. Dans ce cas, des formations sur les conséquences d'un comportement dans un domaine pourraient influencer les comportements d'un autre domaine de la vie.

La question est donc de savoir quelles compétences la formation est-elle censé améliorer, et quel modèle théorique du comportement de conduite automobile sous-jacent oriente les actions et les recherches dans ce domaine. Ainsi, le concept «d'éducation du conducteur » se veut plus large que celui de « formation du conducteur », il l'incorpore et y ajoute d'autres objectifs, contenus et méthodes.

Aux débuts des années 1990, un groupe de chercheurs européens $[12,13]$ a proposé un nouveau modèle théorique du comportement de conduite, dit «modèle hiérarchique » en cinq niveaux, et une matrice d'objectifs pédagogiques correspondant à ce modèle théorique, dite « matrice GDE » (Goals of Driver Education, ou objectifs de l'éducation du conducteur, figure 1). Les objectifs de cette matrice supposent de développer trois types de compétences (des savoirs et des savoir-faire, la compréhension des facteurs de risque qui dégradent le comportement, des compétences d'autoévaluation). On passe ainsi du « contrôle du véhicule » au « contrôle de la personne ». 


\begin{tabular}{|c|c|c|c|}
\hline & $\begin{array}{l}\begin{array}{l}\text { Savoirs } \\
\text { savoir-faire }\end{array} \\
\text { et }\end{array}$ & $\begin{array}{l}\text { Facteurs de } \\
\text { risque }\end{array}$ & Autoévaluation \\
\hline Pressions sociétales, économiques & V.1 & V.2 & V.3 \\
\hline Objectifs existentiels et contrôle de soi & IV.1 & IV.2 & IV.3 \\
\hline Objectifs de la conduite & III.1 & III.2 & III.3 \\
\hline Compréhension des scenarii routiers & II. 1 & II. 2 & II.3 \\
\hline Maîtrise des manœuvres & I.1 & I. 2 & I.3 \\
\hline
\end{tabular}

Fig. 1 La matrice GDE Source [14]

Cette matrice a généré un changement de paradigme, un renouveau des conceptions et des pratiques en Europe ; deux idées deviennent centrales : être capables d'éviter les risques, avoir une évaluation de soi plus réaliste.

La matrice n'est pas un programme de formation au sens traditionnel du terme, elle ne fixe pas de contenus mais décrit des processus (les pressions cybernétiques des niveaux supérieurs de la conduite qui produisent des contraintes d'organisation sur les niveaux inférieurs) et laisse vides, ouvertes les 15 cases des objectifs pédagogiques. Illustrons ce modèle par un exemple : si le sujet présente une certaine vulnérabilité au $5^{\mathrm{e}}$ niveau, celui des pressions sociétales et économiques (par exemple de type sensibilité aux publicités associant grande vitesse et érotisme ou popularité), cette vulnérabilité peut créer un problème au $4^{\mathrm{e}}$ niveau, celui du contrôle de soi (dépendance croissante vis-à-vis de la vitesse pour libérer sa dopamine ou renforcer l'estime de soi), ce problème du $4^{\mathrm{e}}$ niveau produira des contraintes au $3^{\mathrm{e}}$ niveau, celui du rapport à la conduite automobile (posséder un véhicule très puissant et rapide, rechercher des situations routières permettant la grande vitesse), ces contraintes du $3^{\mathrm{e}}$ niveau imposeront des pressions organisationnelles sur les compétences du $2^{\mathrm{e}}$ niveau, celui de la compréhension des scénarios d'accident et des situations de conduite (devoir analyser très rapidement les indices de danger), ces pressions $\mathrm{du}$ second niveau dégraderont enfin les performances du premier niveau, celui de la réussite des manœuvres (ce qui augmentera la probabilité de l'accident lors de l'échec d'une réalisation).

Comme tout phénomène de rétroaction circulaire, les pressions cybernétiques et contraintes organisationnelles peuvent également remonter des niveaux inférieurs vers les supérieurs : par exemple, quand une formation est focalisée sur le pilotage et la maîtrise du premier niveau, ceci peut engendrer des réactions de confiance excessive au quatrième, et annuler les bienfaits préventifs du travail d'entraînement.

Cette vision cybernétique nous permet de comprendre par exemple pourquoi les femmes réussissent moins l'examen $\mathrm{du}$ permis de conduire en première passation mais ont moins d'accidents que les hommes (bien que moins performantes sur les deux niveaux inférieurs de la matrice, elles sont moins déficientes sur les trois niveaux supérieurs), pourquoi des conducteurs peu compétents sur le pilotage peuvent avoir un meilleur bilan accidentel que des conducteurs plus compétents sur ce plan, ou enfin pourquoi certaines formations trop axées sur le premier niveau peuvent se révéler contre-performantes.

La matrice suppose très probablement des partenariats entre divers secteurs car il est peu probable de trouver un formateur qui serait compétent pour «balayer » les 15 cases de la matrice à lui seul ! Si l'on considère les partenariats éducatifs autour de la matrice (les enseignants de l'Éducation nationale, les enseignants de la conduite, les formateurs de formateurs, les psychologues, les examinateurs du permis), on voit que chacun a ses points forts et ses points faibles selon la case de la matrice considérée.

Elle suppose enfin d'impulser pour chaque objectif pédagogique introduit dans les cases une interaction cybernétique décrite par une « figure à 4 coins » (figure 2) : face à un nouvel objectif introduit dans la formation, il faut un formateur compétent, ce dernier doit employer une méthode pédagogique adaptée, et la formation doit être évaluée, testée à la fin du processus.

Lorsque l'on compare les différents niveaux de la matrice, on voit qu'ils diffèrent sur un certain nombre de dimensions, et celles-ci posent des problèmes différents 


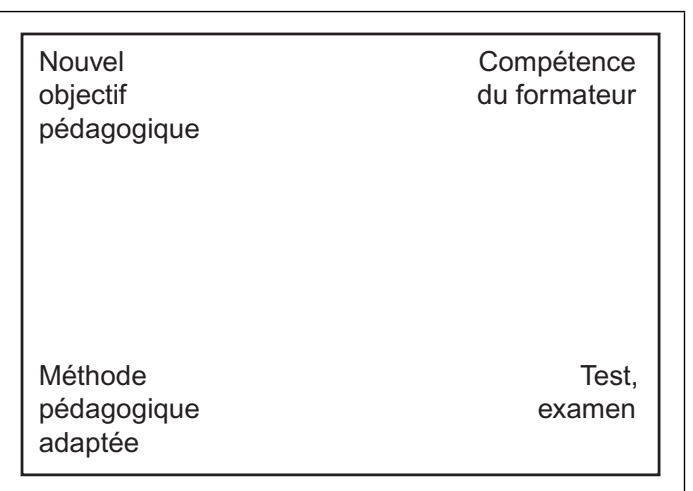

Fig. 2 La «figure des 4 coins» Source [14]

et supposeront des traitements différenciés ; parmi ces dimensions, évoquons :

- le degré de liberté dans l'exécution de la tâche (faible aux premier et second niveaux, fort aux trois niveaux supérieurs : il n'y a pas 36 manières différentes de tourner le volant pour faire un créneau, par contre, il y a de multiples manières de rentrer d'une discothèque...);

- le délai des feedbacks sur son comportement et donc de l'autoévaluation (très courts aux premier et second niveaux, très longs aux trois niveaux supérieurs : lorsque l'on exécute mal une manœuvre de virage, la conséquence est immédiate, par contre, rentrer avec une alcoolémie de $0,7 \mathrm{~g} / \mathrm{l}$ peut se faire pendant des mois, voire des années, avant qu'une conséquence négative se produise, d'où la nécessité des formations post-permis) ;

- le sens de l'information formateur/apprenant : sur les premiers et seconds niveaux, le formateur apprend à l'apprenant des choses qu'il ne sait pas, par contre, sur les trois niveaux supérieurs, c'est l'apprenant qui doit révéler au formateur des choses qu'il ne sait pas, d'où la nécessité des méthodes actives.

Depuis l'élaboration des modèles hiérarchiques du comportement de conduite et de la matrice GDE, divers programmes de formation ont été impulsés et évalués afin d'améliorer la sécurité des jeunes conducteurs novices ; nous nous proposons dans cet article de présenter l'état de la connaissance sur ces questions, en distinguant les formations avant et après le permis. Cette différenciation entre ce qui se fait avant et après le permis peut paraître un peu artificielle et n'est conservée que pour la commodité de l'exposé : des techniques que nous présentons dans la section post-permis (l'entraînement à la perception des dangers, la conduite commentée et même le complément de tâche) sont aujourd'hui préconisées lors de la formation initiale (même si elles sont sans doute rarement mises en œuvre dans des conditions satisfaisantes).

\section{Les formations avant le permis}

Elles concernent le plus souvent les deux niveaux inférieurs de la matrice, concernant la performance (la maîtrise des manœuvres et la compréhension des situations accidentogènes). Elles sont de trois types :

\subsection{La formation par des formateurs professionnels (enseignants de la conduite)}

Cette formation est prépondérante en France et peut être marginale dans d'autres pays ; on estime généralement qu'elle facilite l'obtention du permis, via le succès à l'examen pratique [15]. Les compétences du premier niveau (la maîtrise des manœuvres) sont donc améliorées par cette formation et le gain sécuritaire est obtenu à condition qu'elles ne produisent pas de confiance excessive et d'optimisme comparatif [4]. Ainsi, une évaluation au Danemark des changements intervenus en 1986 dans le programme de formation des conducteurs qui comprenait des formations en classe, de l'entraînement aux manœuvres, de la conduite défensive et de l'entraînement à la perception des dangers [16], a montré quelques effets positifs. L'introduction d'une obligation de formation initiale par des formateurs professionnels avait été introduite avant 1986, mais cette année-là, la formation à la conduite défensive a été introduite dans le programme de formation et dans l'examen du permis. Cette innovation a été associée à une diminution des accidents des conducteurs novices, pour les collisions à plusieurs véhicules mais non pas pour les accidents à un seul véhicule. L'auteur en a conclu que les habitudes de conduite défensive doivent être spécifiques et simples afin d'être aisément enseignées et évaluées, et non pas trop ouvertes à l'interprétation de l'apprenant.

Nous retrouvons ici l'hypothèse de la « figure à 4 coins » : la méthode pédagogique et l'évaluation doivent se correspondre pour voir si les objectifs sont atteints. Si les examens du permis mesuraient ce qu'ils devraient mesurer, ceci soulagerait le travail des formateurs (former pour obtenir l'examen est une obligation inévitable pour les auto-écoles qui sont dans un marché concurrentiel et dont le résultat le plus tangible et important pour leurs clients est d'obtenir le permis...).

En Norvège, la formation par des formateurs professionnels a été associée à des attitudes plus sécuritaires, des infractions moins fréquentes et une diminution de la confiance excessive [17].

De telles évaluations comparant formations professionnelles et non professionnelles n'ont jamais été menées en France, et l'hétérogénéité de la qualité d'un établissement à l'autre serait un facteur à prendre en compte. 


\subsection{Les formations à la conduite en milieu scolaire et universitaire}

Comme indiqué plus haut, les évaluations des formations en milieu scolaire et universitaire ont été plutôt négatives [9] du fait des effets pervers observés.

\subsection{Les formations sur simulateur}

Elles ont été particulièrement développées aux Pays-Bas et sont fondées sur l'idée que le simulateur peut fournir une alternative à l'entraînement pratique sur route en permettant à l'élève de se confronter à des situations d'urgence et à une grande variété de situations routières sans se mettre en danger [18]. Peu de travaux ont évalué l'efficacité de cette stratégie : elle peut améliorer les compétences pratiques, mais il n'y a pas toujours généralisation de l'apprentissage à d'autres compétences dans d'autres contextes, elle peut diminuer le risque accidentel mais uniquement avec les appareils haut de gamme (véhicule complet, champ visuel à $135^{\circ}$ ) [3]. On peut penser en effet que plus le stimulus virtuel se rapproche du réel, meilleure sera l'efficacité, mais ce champ souffre encore de carences méthodologiques importantes (biais de sélection des échantillons et absence de groupes témoins).

\subsection{L'accès graduel à la conduite}

Il ne s'agit pas d'une formation mais d'une réduction de l'exposition au risque, et, globalement, les restrictions imposées par l'accès graduel se traduisent par une diminution de l'implication accidentelle des jeunes conducteurs novices comme nous l'avons évoqué plus haut ; toutefois, la motivation des jeunes apprentis sous-jacente au fait de prendre des cours de conduite a aussi un effet : ainsi, les jeunes conducteurs novices Québécois qui prenaient des leçons afin d'améliorer leurs compétences avaient des taux plus bas d'accidents et d'infractions que ceux dont la motivation était d'accélérer l'accès au permis ou d'obtenir une réduction de la prime d'assurance [19]. Les motivations et les attitudes pourraient donc jouer comme des facteurs de confusion lors des évaluations des programmes de formation.

Les données québécoises de la SAAQ [20] montrent aussi que pour les jeunes conducteurs novices masculins, au moment de leur première passation de l'examen, la combinaison réussite à l'examen théorique/échec à l'examen pratique est associée au plus faible risque d'accident, et la combinaison échec à l'examen théorique/réussite à l'examen pratique est associée au plus fort risque. Ces marqueurs distinguent des sous-groupes de conducteurs qui peuvent se différencier quant aux compétences académiques (capacités de lecture et de compréhension des questions), à la personnalité (préparation consciencieuse de l'examen théorique), aux opportunités de pratiquer la conduite et à l'intérêt porté à la conduite (les principales raisons d'échouer à l'examen pratique étant le manque de pratique et/ou d'intérêt). Ces facteurs se combinent à des différences dans la quantité et la qualité de l'exposition après l'obtention $\mathrm{du}$ permis. Le groupe à haut risque conduit probablement plus et de manière plus dangereuse. Nous devrions donc redéfinir ce que nous appelons « des compétences », la compétence de contrôle d'un véhicule telle qu'elle est mesurée par un test de 30 minutes ne devrait pas être le seul critère de « compétence de conduite ».

\subsection{La conduite accompagnée}

De nouveau, il ne s'agit pas d'une formation mais d'un renforcement de l'expérience de conduite par l'obligation (théorique) des $3000 \mathrm{~km}$ avec le parent accompagnateur, où certes des compétences et des attitudes peuvent être transmises par le «parent formateur». Les principales observations sont désormais bien connues [21]: difficulté de la diffusion de la mesure pour des jeunes dont la situation socio-familiale ne le permet pas ; facilitation de l'obtention du permis $(70 \%$ de réussite en première passation contre $50 \%$ en formation traditionnelle) ; bénéfice préventif sur l'implication accidentelle qui peut être mitigé par divers facteurs : hétérogénéité de la qualité et du respect du cahier des charges d'un établissement à l'autre, absence de formation et de sélection des accompagnateurs, etc.

Il faut en effet rappeler qu'un nombre croissant d'études $[22,23,24]$ montre une association entre les bilans routiers des parents et ceux de leurs enfants. La qualité du conducteur supervisant la conduite accompagnée peut donc être une influence importante sur la qualité des feedbacks correctifs que le conducteur novice peut recevoir.

\section{Les formations après le permis}

Elles peuvent théoriquement concerner tous les niveaux de la matrice et sont de cinq types :

\subsection{Les formations pratiques}

Elles visent à améliorer les compétences techniques (freinage, dérapage, etc.) du premier niveau de la matrice. Certaines évaluations ont montré des effets positifs mais spécifiques et à court terme : par exemple, un programme d'une semaine en Nouvelle-Zélande a amélioré l'adaptation 
du choix de la vitesse mais non pas la perception des dangers ou les attitudes relatives au risque $[25]^{2}$. De même, plusieurs études australiennes ont observé des effets positifs de programmes de deux jours sur le freinage et le contrôle du véhicule, mais là encore sans généralisation de l'apprentissage à divers contextes : par exemple, les jeunes conducteurs novices ayant suivi la formation mettaient plus de temps à freiner sur un véhicule équipé d'ABS que des conducteurs non formés [26].

Parmi les diverses compétences techniques, le dérapage a fait l'objet d'une attention plus particulière, depuis l'abandon de la formation au dérapage dans les pays scandinaves : alors qu'il pouvait sembler logique d'imposer l'obligation de ce type de formation dans ces pays où les routes sont souvent glacées et enneigées, cette obligation a été progressivement abandonnée du fait de ses effets pervers (les conducteurs formés à conduire sur glace présentaient des taux plus élevés d'accidents... sur glace que les conducteurs non formés, du fait du phénomène de confiance excessive) ; ainsi, il a été observé que les jeunes se centraient plus sur la réussite de la manœuvre que sur l'évitement du danger [27] et que cet entraînement accroissait l'écart entre l'autoévaluation de ses compétences en matière de dérapage et ses compétences réelles, ce qui produit l'excès de confiance [28].

Les experts scandinaves ont donc proposé l'abandon de cette stratégie ou sa réforme, par exemple en centrant l'entraînement sur l'évitement du danger et en structurant les exercices de manière à ce qu'ils ne puissent être réussis à des vitesses dangereuses (certains exercices en Suède consistent par exemple à mettre le jeune en échec afin qu'il réduise sa vitesse de lui-même, améliorant ainsi l'autoévaluation ; à la différence des formations nord-américaines, on provoque les pertes de contrôle à faible vitesse, ainsi on n'effraie pas et ne sape pas la confiance des timides) ; toutefois, même en focalisant sur l'évitement du danger, des évaluations ultérieures Katila et al. [29] ont montré un décalage entre les conceptions des formateurs (l'évitement) et celles des jeunes (attente de maîtrise). Par ailleurs, ces évaluations de la réforme de l'entraînement au dérapage ont montré des diminutions des taux d'accidents pour les conducteurs de plus de 21 ans, mais des augmentations des taux d'accidents pour les conducteurs de moins de 21 ans ; ces observations sont liées à la question âge/expérience et supportent l'idée que le timing des interventions éducatives doit être un problème auquel porter attention.

Enfin, une évaluation randomisée a été réalisée dans le Montana à propos des effets d'une formation d'une journée à la conduite défensive (manœuvres d'urgence, dérapage,

\footnotetext{
${ }^{2}$ Mais sur une population de jeunes conducteurs encore dans la phase restrictive du permis.
}

contrôle du véhicule) sur 347 jeunes conducteurs novices [30]; un suivi sur 4 ans ne montre pas d'effets sur les accidents ou les infractions.

\subsection{Les formations cognitives}

Elles visent à améliorer les compétences nécessaires au second niveau de la matrice, l'anticipation et la compréhension des situations routières dangereuses et des scénarii d'accidents.

\subsubsection{L'entrâ̂nement à la perception des dangers}

Cette stratégie a été particulièrement développée en Angleterre du fait des nombreux travaux menés dans ce pays sur les relations entre perception des dangers et risque d'accident : en effet, les conducteurs novices ont des moins bonnes compétences dans ces domaines que les conducteurs expérimentés, leurs explorations visuelles sont moins efficaces, ils regardent moins les rétroviseurs extérieurs, et leur champ de vision périphérique est plus réduit [31]. Les experts anglais ont donc développé des tests de perception des dangers à partir de vidéos où l'élève doit détecter des indices de danger et prendre des décisions, et montré que les résultats de ces tests étaient prédictifs de l'implication accidentelle lors de la première année de la conduite [32].

De nombreux programmes en vidéo, avec arrêt sur image et questionnement «Que va-t-il se passer maintenant?» ont été développés et montrent autant d'effets sur les conducteurs novices que sur les expérimentés, sur les infractionnistes que sur les non infractionnistes; ils sont particulièrement utiles pour les dangers qui surgissent brusquement, peu entraînables en pratique, et pour les dangers cachés pour les novices.

La question est donc de savoir si l'entraînement à la perception des dangers peut améliorer cette compétence ; deux types différents de formation ont été évalués :

\section{i. la conduite commentée}

Cette formation consiste à fournir des commentaires aux élèves lorsqu'ils conduisent ou lorsqu'ils regardent une vidéo ; les jeunes conducteurs qui ont suivi cette formation ont de meilleurs scores aux tests de perception des dangers, améliorent leurs explorations visuelles périphériques et réduisent plus leurs vitesses lorsqu'ils approchent d'un danger dans les tests sur simulateur $[33] .^{3}$

\footnotetext{
${ }^{3}$ Mais sur une population de 36 participants sur 40 qui n'avaient pas encore passé l'examen ; on peut néanmoins penser que la conduite commentée pourrait être utile après l'examen...
} 
Cette approche améliore le respect des distances de sécurité chez les conducteurs formés, ceci peut être considéré comme une amélioration des comportements dangereux et infractionnistes, du moins de ceux qui sont liés à l'inexpérience et aux dysfonctionnements de la perception du danger (et non pas de ceux qui sont liés à l'âge et aux prises de risque délibérées, $c f$. Assailly [4]). Une approche similaire a consisté à exposer les jeunes conducteurs à une série de situations dangereuses sur simulateur, puis l'élève formé visionne de nouveau une vidéo de sa performance en la comparant à celle d'un expert [34]. Cet entraînement améliore l'anticipation des dangers.

ii. le complètement de tâche

Dans cette approche, les participants doivent regarder des vidéos et compléter les tâches comme prédire les dangers possibles ou indiquer des actions appropriées ; les sujets formés ont des performances améliorées, leurs explorations visuelles sont meilleures (ils fixent plus les zones de danger potentiel, que ce soit sur simulateur ou sur route [35]).

Ces résultats sont donc encourageants mais, comme pour la conduite commentée, nous manquons de données pour savoir si ces progrès se traduisent réellement en termes d'accidents.

L'entraînement à la perception des dangers conduit donc à la comparaison de différents types de formation : programme simple en vidéo, arrêt sur image, "Que va-t-il se passer maintenant? »; programme de conduite commentée par expert ; programme hybride de conduite commentée par expert/sujet ; programme complet (vidéo plus hybride). Les quatre formations peuvent améliorer la perception des dangers, le moins efficace étant la vidéo simple, le plus efficace le programme complet.

Enfin, nous devons considérer le timing en fonction de l'âge et de l'expérience (un jeune ne maîtrisant pas encore le premier niveau de la matrice ne profitera pas de ces entraînements), le transfert d'apprentissage d'une situation de formation à l'autre, et la faisabilité (économique, logistique) de ce transfert. Il nous manque pour le moment sur ce sujet des suivis plus longs et de savoir qui en a le plus besoin parmi les jeunes conducteurs novices.

Pour conclure, sur ces formations post-permis, nous pouvons dire que les formations pratiques améliorent les compétences techniques mais que le gain de sécurité n'est pas évident car les jeunes conducteurs novices ne vont pas ensuite utiliser correctement ces savoir-faire, et que les formations cognitives semblent bien améliorer la prise de conscience des indices de danger (et ceci plus pour des conducteurs novices que pour des conducteurs expérimentés) sans que des données probantes sur l'implication accidentelle soient encore disponibles.

\subsection{Les formations sur les compétences de vie}

Plus récemment sont apparues des formations visant le quatrième niveau de la matrice, c'est-à-dire le développement de compétences visant la personne et non plus le conducteur, intitulées « compétences de vie » (life skills) : il s'agit, entre autres, du renforcement de l'estime de soi, de l'assertivité ou affirmation de soi en groupe, des capacités de résistance aux pressions, du contrôle de soi, de l'efficacité perçue de soi, etc. On peut penser aussi aux compétences dites « métacognitives » telles que l'autoévaluation. Bref, ces formations n'ont plus aucun contenu en rapport direct avec la tâche de conduite d'une voiture.

Deux exemples récents de tels programmes en milieu scolaire, évalués ou en cours d'évaluation :

- le programme You Hold the Key (YHTK) développé dans l'Ohio en direction des conducteurs adolescents de 15-19 ans (qui sont déjà conducteurs de voiture dans ce pays) ; il dure dix semaines et comporte une grande variété d'activités : des présentations Powerpoint, des interventions de policiers et de juges sur l'infraction-alcool, des panels de discussion avec des jeunes adultes, des témoignages de victimes, des vidéos sur diverses thématiques de l'insécurité routière des jeunes ;

- le programme ESPACE à Poitiers [36] visant à favoriser une consommation d'alcool socialement responsable, par deux objectifs (retarder l'âge de début de la consommation régulière d'alcool ; retarder l'âge de début des ivresses), en direction de 1200 collégiens. Il est centré sur l'amélioration des compétences de vie, de l'autoévaluation et de l'estime de soi, et il utilise des jeux de rôle et des discussions de groupe.

Ces approches de life skills sont donc holistiques et s'adressent globalement à la santé et au bien-être des jeunes, car elles posent que les états de détresse psychologique sont bien souvent au cœur de la genèse des phénomènes de prise de risques, d'addiction et de transgression (Assailly, 2007) [37]. Leur difficulté réside dans le temps, l'investissement et l'engagement qu'elles supposent, ce qui limite pour le moment le nombre de tentatives.

\subsubsection{Les entraînements des « compétences de vie »}

Les évaluations menées aux États-Unis et en Australie ont conclu à des effets positifs de telles formations : augmentation du taux de port de la ceinture, de la capacité d'éviter les situations de conduite sous influence par le programme You Hold the Key [38], diminution des taux d'accidents par un programme d'intervention 
communautaire en Australie [39]. En France, le programme ESPACE a déjà montré des impacts positifs sur l'estime de soi et les compétences de vie (capacité de résister à la pression du groupe) des 11/13 ans.

L'influence des passagers sur le conducteur a fait l'objet de programmes d'entraînement en Australie (apprendre à mieux communiquer vers le conducteur, à influencer le conducteur) ; on considère l'ensemble conducteur-passager comme une « équipe » et on applique les concepts venant de la sécurité de l'aviation, des plateformes pétrolières, des contrôleurs aériens et des professions de santé, les crew ressources management dans les situations critiques (cognitions partagées, conscience de la situation distribuée, modèles mentaux partagés). Donc l'influence des passagers peut ne pas être négative et ce serait particulièrement important pour les jeunes conducteurs novices les premières années de la conduite.

\subsubsection{Les programmes d'amélioration de l'autoévaluation (diminution de la confiance excessive ou insight)}

Deux méthodes usitées consistent à demander au sujet de se comparer à un conducteur « moyen », ou bien de comparer son autoévaluation à celle d'un instructeur ; on peut ainsi dégager la prévalence de la confiance excessive ou de l'optimisme comparatif : de 30 à $40 \%$ des jeunes conducteurs novices se surestiment, $50 \%$ s'évaluent correctement et 10 à $20 \%$ se sous-estiment [40,41]; donc, nous voyons que tous les jeunes conducteurs novices ne nécessitent pas une amélioration de l'autoévaluation et une diminution de la confiance excessive !

Comme évoqué plus haut, l'entraînement avec des formateurs professionnels semble réduire l'excès de confiance ; celle-ci diminue d'ailleurs avec l'expérience de conduite : ce sont les conducteurs très inexpérimentés qui se surestiment fortement, puis, avec l'expérience, les autoévaluations suivent les progrès des compétences [42,43] (sur des apprentis en formation initiale).

Des programmes d'amélioration de l'autoévaluation (ou d'insight) pourraient donc diminuer la confiance excessive, des évaluations ont apporté des résultats prometteurs [28] mais des carences méthodologiques importantes subsistent.

\subsection{Le coaching}

Comme évoqué plus haut, l'amélioration de l'autoévaluation et les niveaux supérieurs de la matrice supposent des échanges entre le formateur et l'apprenant ; à cette fin, un projet européen [44] a développé des scénarios de coaching tels que 1. l'esquisse commune (l'apprenant esquisse comment il va gérer une situation routière), 2. l'apprenant devient 1'enseignant, 3. comparer et contraster (pour les distances de sécurité), 4. renforcement positif : réfléchir sur les raisons des choix, 5. rétroaction par vidéo : on filme la leçon de conduite puis on regarde ensuite pour analyser. On voit bien comment ces scénarios permettent d'améliorer la communication formateur/apprenant.

\subsection{Les secondes phases de formation}

Les secondes phases de formation ont été développées dans huit pays européens (Suisse, Autriche, Luxembourg, Finlande, Estonie, Slovénie, Lituanie et Croatie). Elles combinent généralement sur deux jours exercices sur circuit, sur route et en salle. En Autriche, on leur attribue une diminution des accidents et des infractions des jeunes conducteurs (Myntinnen et al., 2010) [45]; en Finlande, des effets positifs ont été observés mais seulement sur la conduite économique (Myntinnen et al., op. cit.) [46]. Toutefois, des débats subsistent sur les vérifications statistiques de la validité des effets de ces formations.

\section{Conclusion}

La mise en évidence d'un impact d'une formation sur le risque accidentel ultérieur des jeunes conducteurs novices n'apparaît pas comme évidente du fait de multiples difficultés d'ordre méthodologique (manque fréquent de groupe témoin, de contrôle des biais de sélection des échantillons, de suivi suffisamment long et précis sur les accidents et les infractions, etc.) ; par contre, des améliorations du comportement de conduite ont pu être observées. Certains types de formation (par exemple, les formations au pilotage) améliorent les compétences sans diminuer le risque, d'autres types de formation (par exemple, les entraînements aux « compétences de vie ») diminuent le risque sans influencer les compétences.

Il ne s'agit pas d'opposer mais de réunir ces deux observations au sein de la formation : il faut apprendre aux conducteurs novices à éviter le danger, mais aussi à savoir l'affronter quand ils ne peuvent l'éviter.

Par ailleurs, les différents types de formation n'ont pas les mêmes effets en fonction de leur situation dans le temps au sein de l'acquisition de l'expérience : un gain acquis avant le permis peut très bien ne pas perdurer après le permis car les contextes de la conduite ont changé. En effet, l'apprentissage ne se réduit pas à la formation initiale : le jeune conducteur novice continue à apprendre par lui-même après le permis grâce à l'expérience et l'exposition ; certaines compétences ont d'ailleurs 
des courbes rapides d'apprentissage après le permis (la maîtrise des manœuvres, notamment par le phénomène de l'automatisation de celles-ci), d'autres compétences ont des courbes bien plus lentes (l'éco-conduite, l'attention portée aux usagers vulnérables par exemple). On connaît bien aussi le phénomène du relâchement du respect des règles et des procédures au bout des six mois après l'obtention du permis.

Avant le permis, il semble donc important de ne pas se focaliser seulement sur les compétences pratiques mais d'insister sur le risque et les compétences de vie nécessaires pour éviter le risque. Après le permis, il s'agit de lutter contre la confiance excessive, en développant la perception des dangers et les compétences métacognitives (autoévaluation plus réaliste de soi).

À partir des réflexions actuelles des formateurs [46,47] à propos de la matrice et de sa traduction institutionnelle, le REMC (Référentiel pour l'éducation à une mobilité citoyenne, 2013, qui est devenu le programme officiel de la formation), diverses conséquences pédagogiques ont été avancées :

- il faut toujours associer, alterner et combiner apprentissages théoriques et pratiques, afin que l'apprenti conducteur comprenne les raisons des règles et procédures ;

- il faut toujours partir des problèmes de compréhension de l'apprenant (ses erreurs sur les distances de sécurité, ses prises d'information, son adaptation de sa vitesse au contexte) afin d'améliorer son autoévaluation ;

- il faut travailler sur l'expérience, l'accumulation des kilomètres ne suffit pas, il faut améliorer la diversité des situations rencontrées et la capacité d'analyser ces situations ;

- dans le cadre de la conduite accompagnée, il faut augmenter le plus possible le nombre d'heures de conduite supervisée, avant et après le permis.

Une refondation future de la formation initiale des jeunes conducteurs novices à partir des travaux sur la matrice est en projet en France, sous l'acronyme du CECUR (Continuum éducatif du citoyen usager de la route) dans un premier temps pour le permis voiture. Ce futur cursus apporte une innovation importante : une progressivité vers la validation du permis de conduire, l'examen pratique devenant une étape de la formation, et non plus sa clôture et objectifs ultimes. Avant la passation de l'examen, les apprentis conducteurs participeraient à un suivi de cinq ateliers de gestion des risques en salle et en groupe, et après l'obtention du permis de conduire à un suivi de deux rendez-vous d'expériences, l'objectif est de travailler sur le partage des expériences vécues par chacun pour s'approprier durablement les comportements sécuritaires. Ces deux composants de la formation ont pour objectif d'améliorer l'autoévaluation et d'atteindre les niveaux supérieurs de la matrice.
Si nous disposons d'une imposante littérature sur les actions de formation qui s'adressent aux deux premiers niveaux de la matrice, c'est-à-dire qui essaient d'améliorer la performance de conduite, bien plus rares sont les données qui existent concernant les trois niveaux supérieurs de la matrice, c'est-à-dire qui essaient d'améliorer la motivation à se comporter de manière sécuritaire.

$\mathrm{Au}$ troisième niveau, nous pouvons néanmoins mentionner les travaux sur la formation à l'éco-conduite (notamment compilés à la CIECA, à l'INSERR, etc.), les initiatives sur l'éco-mobilité, les travaux sur les alternatives à la conduite sous l'influence de l'alcool (actions de sensibilisation en discothèques ou en milieu scolaire, telles que les « lunettes d'alcoolémie », désignation d'un conducteur, prévention par les pairs, etc.).

Aux quatrièmes et cinquièmes niveaux de la matrice, beaucoup reste encore à faire, que ce soit au niveau des actions que de leurs évaluations. Nous avons exposé ici les diverses approches pédagogiques en les répartissant entre avant et après le permis, mais en fait, intégrer les quatrième et cinquième niveaux de la matrice dans la formation à la conduite supposera, comme le prévoit le CECUR, de faire descendre en formation initiale des méthodes qui sont utilisées en post-permis (comme par exemple les jeux de rôle, les discussions de groupe, les questionnaires d'autoévaluation, etc.) pour améliorer l'autoévaluation des conducteurs novices à propos de leurs problèmes de vitesse, d'alcool, de cannabis, de téléphone, de stress, etc. Nos futurs travaux évaluant les actions de sensibilisation menées actuellement dans les lycées devraient par exemple apporter des éléments de réflexion, et de nombreuses autres recherches pourraient être menées dans ce domaine, notamment sur l'appariement entre type de conducteur et type de programme éducatif ou préventif.

Même s'il reste beaucoup à réfléchir et à construire, nous avons vu que les évolutions en France sont en lien avec un corpus de recherches et de connaissances bien défini mais s'inscrit aussi dans le cadre d'une évolution continue et progressive plus longue (le PNF, l'enseignant de la conduite plutôt que le moniteur, le continuum éducatif, l'AAC, etc.). L'approche par le REMC et les compétences mais aussi les évolutions actuelles (titre professionnel) ou futures (BAFM, CECUR) en sont les fruits les plus récents, notamment avec l'accent mis dans les programmes, pour diminuer l'occurrence des accidents, sur la prise de conscience et la compréhension des risques en complément de la maîtrise des situations de conduite.

Pour conclure, les différences exposées au début de cet écrit entre approches visant la diminution de l'exposition au risque et celles visant l'éducation sont en train de s'amenuiser et la matrice GDE pourrait à ce titre être un outil permettant une forme de synthèse. Enfin, tout ce travail réalisé par l'éducation routière autour de la matrice 
pourra être utile à d'autres secteurs d'intervention dans notre société (santé, délinquance, éducation inclusive, etc.) tant on voit bien que les mécanismes mis à jour par la matrice sont à l'œuvre dans bien d'autres aspects que le comportement routier.

\section{Bibliographie}

1. ONISR (2015) La sécurité routière en France. Bilan de l'accidentalité de l'année 2014, La Documentation Française.

2. Assailly, JP (2001). La mortalité des jeunes, PUF, coll. « Que sais je? », Paris.

3. Allen, RW, Park, GD (2007). The effect of driving simulator fidelity on training effectiveness. In: Proceedings of the Driving Simulator Conference North America, Iowa City.

4. Assailly, JP (2010). La psychologie du risque. Lavoisier, coll. « Sciences du risque et de la décision », Paris.

5. Assailly, JP (2016) La mortalité des jeunes, Codes Rousseau.

6. Mayhew, DR, Simpson, HM, Pak, A (2003) Changes in collision rates among novice drivers during the first months of driving, Accident Analysis \& Prevention 35: 683-691.

7. Waller, PF (2003) The genesis of GDL, Journal of Safety Research 34(1), 30: 17-23.

8. Russell, KF, Vandermeer, B, Hartling, L (2011) Graduated driver licensing for reducing motor vehicle crashes among young drivers. Cochrane Database Syst. Rev. 10: art. No.: CD003300.

9. Lund, AK, Williams, AF, Zador, P (1986). High school driver education: further evaluation of the DeKalb County study, Accident Analysis \& Prevention 18: 349-357.

10. Smith, MF (1994) Research agenda for an improved novice driver education program . Report $\mathrm{n}^{\circ}$ DOT HS 808 161. office of program development and evaluation, traffic safety program, NHTSA, Washington, D.C. 20590.

11. Shell, DF, Newman, IM (2015) Driver education and teen crashes and traffic violations in the first two years of driving in a graduated licensing system, Accident Analysis \& Prevention 82: 42-52.

12. Siegrist, S (Ed.) (1999) Driver training, testing and licensingtowards theory-based management of young drivers' injury risk in road traffic. Results of EU-Project GADGET, work package 3. BfuReport 40. SchweizerischeBeratungsstelleFuerUnfallverhuetung, Berne.

13. Hatakka, M, Keskinen, E, Gregersen, NP, Glad, A, Hernetkoski, K (2002) From control of the vehicle to personal self-control: broadening the perspectives to driver education, Transportation Research Part F: 201-215.

14. Assailly, JP (2013) Quelles innovations pédagogiques dans le cadre de la réforme du permis de conduire ? Questions Vives, recherches en éducation, 9(19) « Éducation routière, changement de comportement et formation à la conduite »: 37-50.

15. Nyberg, A, Gregersen, NP, Wiklund, M (2007). Practicing in relation to the outcome of the driving test, Accident Analysis \& Prevention 39: 159-168.

16. Carstensen, G (2002) The effect on accident risk of a change in driver education in Denmark, Accident Analysis \& Prevention 34: 111-121.

17. Tronsmoen, $T$ (2010) Associations between driver training, determinants of risky driving behaviour and crash involvement. Safety Science 48: 35-45.

18. De Winter, JCF, Wieringa, PA (2007) Violations and errors during simulator-based driver training. Ergonomics 50: 138-158.

19. Hirsch, P, Maag, U, Laberge-Nadeau, C (2006) The role of driver education in the licensing process in Quebec, Traffic Injury Prevention 7: 130-142.
20. Laberge-Nadeau, C, Maag, U, Bourbeau, R, Desjardins, D, Messier, S, Hirsch, P (1999) Le lien entre la performance aux examens (théorique et pratique) pour l'obtention d'un permis et le taux d'implication dans les accidents. Rapport de recherche CRT-99-56, Centre de recherche sur les transports, Université de Montréal, 144 pages.

21. Page, Y, Ouimet, MC, Cuny, S (2004) Estimation du risque relatif d'implication accidentelle des jeunes conducteurs ayant suivi l'Apprentissage Anticipé de la Conduite, Rapport CEESAR/Fondation MAIF.

22. Ferguson, SA, Williams, AF (2001) Relationship of parent driving records to the driving records of their children, Accident Analysis \& Prevention 33(2, 1): 229-234.

23. Bianchi, A, Summala, H (2004) The "genetics" of driving behavior: parents' driving style predicts their children's driving style, Accident Analysis and Prevention 36: 655-659.

24. Taubman-Ben-Ari, O, Kaplan, S (2015) Parents'and peers' contribution to risky driving of male teen drivers, Accident Analysis \& Prevention 78: 81-86.

25. Isler, RB, Starkey, NJ (2011) Effects of higher-order driving skill training on young, inexperienced drivers' on-road driving performance, Accident Analysis \& Prevention 43: 1818-1827.

26. Petersen, A, Barrett, R (2006) Driver-training and emergency brake performance in cars with antilock braking systems, Safety Science 44: 905-917.

27. Katila, A, Keskinen, E (1996) Conflicting goals of skid training, Accident Analysis \& Prevention 28: 785-789.

28. Gregersen, NP (1996) Young drivers' overestimation of their own skill - an experiment on the relation between training strategy and skill, Accident Analysis \& Prevention 28: 243-250.

29. Katila, A, Keskinen, E (2004) Does increased confidence among novice drivers imply a decrease in safety? The effects of skid training on slippery road accidents, Accident Analysis \& Prevention 36: 543-550.

30. Kelly, MJ (2005) Effects of Defensive Vehicle Handling Training on Novice Driver Safety: Phase 1. Preparation for Advanced Driver Training. Western Transportation Institute, Bozeman, MT

31. Underwood, G, Crundall, D (2002) Selective searching while driving: the role of experience in hazard detection and general surveillance. Ergonomics 45: 1-12.

32. Boufous, S, Ivers, R (2011) Attempts at the practical on-road driving test and the hazard perception test and the risk of traffic crashes in young drivers. Traffic Injury Prevention 12: 475-482.

33. Crundall, D, Andrews, B (2010) Commentary training improves responsiveness to hazards in a driving simulator, Accident Analysis \& Prevention 42: 2117-2124.

34. Wang, Y, Zhang, W (2010) Effects of a simulation-based training intervention on novice drivers' hazard handling performance. Traffic Injury Prevention 11: 16-24.

35. Fisher, DL, Pollatsek, AP (2006) Can novice drivers be trained to scan for information that will reduce their likelihood of a crash? Injury Prevention 12: i25-i29.

36. Bailly, D, Rouchaud, A (2015) Consommation d'alcool chez les jeunes adolescents : enquête en milieu scolaire. Alcohol use in young adolescents. A survey in French secondary schools, Archives de Pédiatrie 22: 510-517.

37. Assailly, JP (2007) Jeunes en danger. Les familles face aux conduites à risques, Imago, Paris.

38. King, KA, Vidourek, RA (2008) Teaching adolescents safe driving and passenger behaviors: effectiveness of the You Hold the Key Teen Driving Countermeasure, Journal of Safety Research 39: 19-24.

39. Senserrick, T, Ivers, R (2009) Young driver education programs that build resilience have potential to reduce road crashes, Pediatrics 124: 1287-1292. 
40. Mynttinen, S, Sundström, A (2009a) Are novice drivers overconfident? A comparison of self-assessed and examiner-assessed driver competences in a Finnish and Swedish sample, Transportation Research Part F, Traffic Psychology Behavior 12: 120-130.

41. Mynttinen, S, Sundström, A (2009b) Self-assessed driver competence among novice drivers-a comparison of driving test candidate assessments and examiner assessments in a Dutch and Finnish sample, Journal of Safety Research 40: 301-309.

42. Boccara, V, Delhomme, P (2011a) Development of student drivers' self-assessment accuracy during French driver training: self assessments compared to instructors' assessments in three risky driving situations, Accident Analysis \& Prevention 43: 1488-1496.

43. Boccara, V, Delhomme, P (2011b) Time course of driving-skill self-assessments during French driver training, Accident Analysis \& Prevention 43: 241-246.
44. HERMES project (2010) State-of-the-Art report on "Coaching and optimal communication skills" for driving instructors in the context of category $B$ driver training, www.gutefahrt.at/hermes.

45. Mynttinen, S., Gatscha, M (2010) Two-phase driver education models applied in Finland and in Austria-Do we have evidence to support the two phase models? Transportation Research Part F: Traffic Psychology and Behaviour 13(1): 63-70.

46. Hernja, G (2007) Approche cognitive des comportements des élèves des écoles de conduite face aux situations d'apprentissage, Perspectives de recherches pour faire évoluer les systèmes de formation. Recherche Transports Sécurité 97: 271-282.

47. Hernja, G, Higelé, P (2008) La compréhension des situations de conduite et les prises de risques chez les conducteurs novices jeunes, Recherche, Transports, Sécurité 98: 13-37.

48. De Winter, JCF, de Groot, S (2009) Relationships between driving simulator performance and driving test results. Ergonomics 52: $137-153$. 